\title{
Periodic orbit quantization of chaotic systems with strong pruning
}

\author{
Kirsten Weibert, Jörg Main, and Günter Wunner \\ Institut für Theoretische Physik 1, Universität Stuttgart, D-70550 Stuttgart, \\ Germany
}

\begin{abstract}
The three-disk system, which for many years has served as a paradigm for the usefulness of cycle expansion methods, represents an extremely hard problem to semiclassical quantization when the disks are moved closer and closer together, since (1) pruning of orbits sets in, rendering the symbolic code incomplete, and (2) the number of orbits necessary to obtain accurate semiclassical eigenvalues proliferates exponentially. In this note we show that an alternative method, viz. harmonic inversion, which does not rely on the existence of complete symbolic dynamics or other specific properties of systems, provides a key to solving the problem of semiclassical quantization of systems with strong pruning. For the closed three-disk system we demonstrate how harmonic inversion, augmented by a signal cross-correlation technique, allows one to semiclassically calculate the energies up to the 28 th excited state with high accuracy.
\end{abstract}

\section{Introduction}

The question as to the connection between quantum mechanics and classical dynamics lies at the heart of physics, and therefore has attracted continual attention for several decades. A milestone for understanding this relation in chaotic systems was Gutzwiller's trace formula [1], which provided the semiclassical approximation to the quantum density of states in terms of a sum over all periodic orbits of the corresponding classical system. However, it is a well known, and fundamental, problem of the trace formula that it does not normally converge in the physical energy domain, mainly as a consequence of the rapid proliferation of periodic orbits with growing period. Various techniques have been designed to overcome this problem, though most of them rely on special properties of the individual systems in point, such as ergodicity and the existence of a complete symbolic dynamics [2-4]. As an alternative, the method of harmonic inversion has been developed and proven [5-7] in recent 
years to be universal in scope as regards the extraction of semiclassical eigenvalues from trace formulae, in the sense that (1) it can be applied to both chaotic and regular systems and (2) does not require any specific properties of the system.

It is the purpose of this Letter to show that harmonic inversion also provides a key to solving the problem of semiclassical quantization of systems with symbolic dynamics that exhibit strong pruning. As a prototype of such systems, we choose the three-disk billiard, and demonstrate that even in the particularly challenging limit of mutually touching disks the method allows us to semiclassically calculate the energy eigenvalues up to the 28th excited state with high accuracy.

The three-disk billiard system consists of three equally spaced hard disks of unit radius. Large distances $d$ between the centers of the disks, especially $d=6$, have been studied as a test case for periodic orbit quantization of chaotic systems in a number of investigations in recent years. In particular, the system has served as a model for the utility of the cycle expansion method [2,8-10]. At large distances between the disks, the dynamics of the system is completely hyperbolic, and it is possible to uniquely label the orbits by a complete symbolic code. Also, at large distances, the basic assumption of the cycle expansion, namely, that the contributions of long periodic orbits are shadowed by contributions of corresponding combinations of short orbits, is very well fulfilled. The actions and stability coefficients of the orbits are essentially determined by the cycle length, and the number of orbits up to a given action is relatively small. The situation, however, changes completely when the disk separation is reduced: As for decreasing $d$ all orbits become shorter, the total number of orbits up to a given value of the action increases rapidly. Moreover, the parameters of the orbits turn out to be no longer simply determined by the cycle length, the condition of shadowing is more and more badly fulfilled, and, finally, at $d=2.04821419$, pruning sets in [11], i.e., part of the periodic orbits becomes unphysical in that they run through one of the disks, and have to be discarded, rendering the symbolic code incomplete. The number of pruned orbits grows rapidly, as the distance between the disks is further decreased. Finally, in the limiting case of touching disks, $d=2$, the system exhibits strong pruning, and the conditions for the convergence of the cycle expansion are no longer satisfied at all.

A remarkable first step towards semiclassical quantization of the closed threedisk system was achieved by combining the conventional cycle expansion with a functional equation [12]. Based on periodic orbit corrections to the mean density of states, approximations to the lowest eigenvalues were obtained from a small set of periodic orbits up to cycle length $l=3$, for which the symbolic dynamics is still complete (including formally a zero length orbit). 

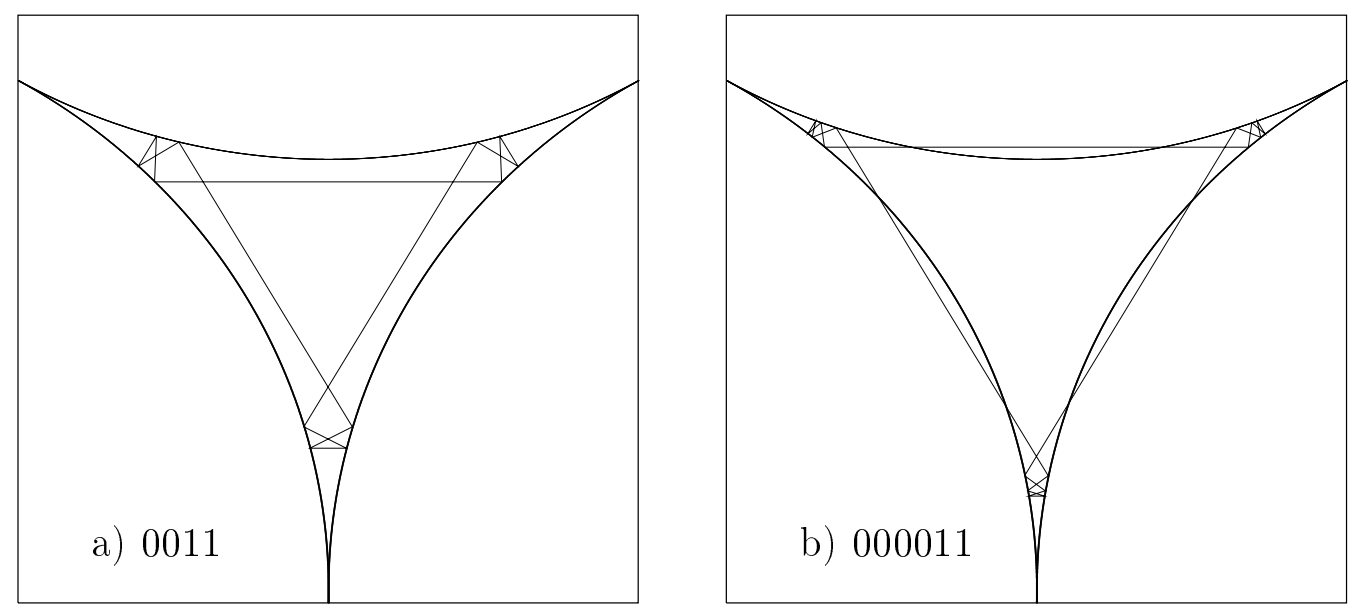

Fig. 1. Two examples of periodic orbits in the closed three-disk system (full domain representation). Orbit a) is physical, while orbit b) runs through the disks and therefore has to be pruned. For both orbits, the symmetry reduced symbolic code is given.

It is our aim in this Letter to dispose of special additions to semiclassical quantization, like functional equations, and apply "pure" periodic orbit quantization by including longer orbits even in regions where strong pruning occurs. The method applied here to the particular example of the closed three-disk billiard will be the key for the semiclassical quantization of a large variety of other challenging systems lacking special structural information on the quantum or classical level such as the existence of a complete symbolic dynamics or the applicability of a functional equation.

\section{Periodic orbits of the closed three-disk system}

In the numerical search for periodic orbits, we can make use of a symmetry reduction characteristic of this system [2], i.e., periodic orbits are calculated in a fundamental domain and labeled by a binary code with the alphabet ' 0 ' and ' 1 '. In the case of touching disks, the orbits can be grouped in "channels" with the same "tail" (end letters) but increasing number of leading zeros in the symbolic code. These orbits have the same basic geometric shape but run deeper and deeper into the corner formed by two touching disks, bouncing back and forth between the two disks, until finally - with only two exceptions - the channel breaks off because the orbits become pruned. An example is shown in Fig. 1. Adding a leading zero to the code increases the action of the corresponding orbit only slightly. This means that there is a huge number of orbits with very long symbol lengths but relatively small, similar, actions.

We searched for periodic orbits of the closed three-disk system with physical 


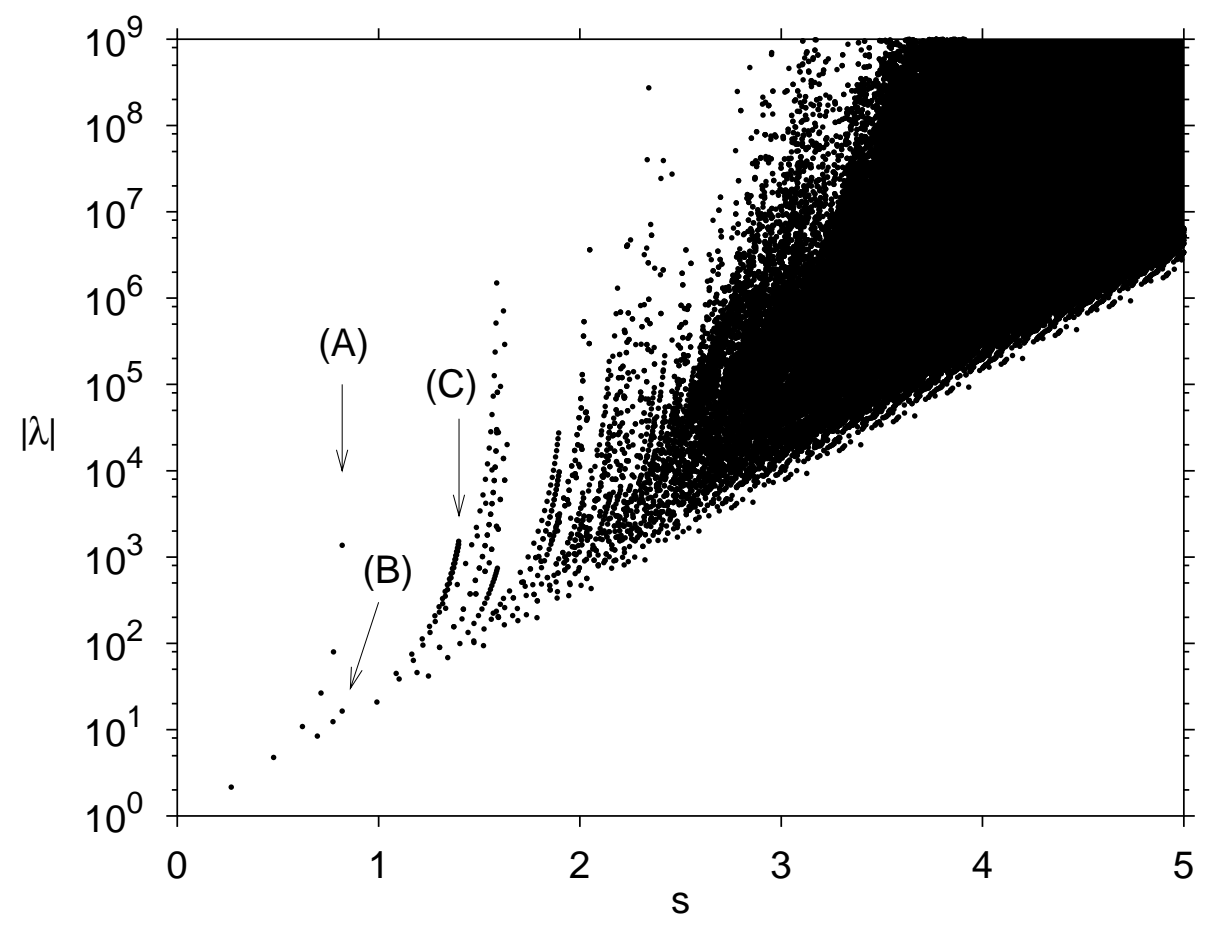

Fig. 2. Distribution of periodic orbit parameters of the closed three-disk system. The quantities plotted are the absolute value of the larger stability eigenvalue $|\lambda|$ versus the physical length $s$ of the orbits. For clarity, a few channels of orbits are marked by arrows. Channels (A) and (B) break off because of pruning. The two channels marked $(\mathrm{C})$ in fact contain infinitely many orbits, but have been cut off by restricting the maximum number of successive zeros in the symbolic code (see text).

length $s<s_{\max }=5.0$. The number of orbits proliferates very rapidly with increasing length. Therefore, it is impossible in practice to determine all orbits up to this length, but one has to introduce reasonable restrictions for the orbits to be included. The orbits were calculated channel by channel, starting from the shortest orbit in the channel and adding more and more leading zeros to the code. As the orbits become more unstable with increasing length of the symbolic code, the main contributions to Gutzwiller's trace formula arise from the shortest orbits of each channel. Therefore, we stopped the calculation of orbits in a given channel when the absolute value of the larger eigenvalue $\lambda$ of the monodromy matrix exceeded a set value of $\lambda_{\max }=10^{9}$. Furthermore, to make the calculations feasible, the maximum number of successive zeros in the code was restricted to 20 for orbits with length $s<3.1$, and to 12 for orbits with $3.1<s<5.0$. The final set of orbits obtained by an extensive numerical periodic orbit search consists of about 5 million primitive periodic orbits, and is illustrated in Fig. 2, where each dot in the diagram marks the parameter pair $(s,|\lambda|)$ of a primitive periodic orbit. In the region $s \lesssim 2.5$ various channels are clearly distinguishable, a few of which are indicated by arrows. As mentioned above, most of the channels break off because of pruning. 
However, the number of orbits grouped in a channel can be very large or, for the two channels labeled (C), even infinite. [The cutoff for (C) in Fig. 2 is caused by restricting the number of successive zeros in the symbolic code as explained above.] Note that the total number of orbits in those channels which break off because of the restriction of the number of successive zeros in the symbolic code is usually much larger than in those channels where the number of orbits is physically restricted by pruning, which means that pruning is the dominating effect for the cutoff of orbits in the various channels.

\section{Periodic orbit quantization by harmonic inversion}

The task, and challenge, now is to extract semiclassical eigenvalues in a numerically stable way from the huge set of periodic orbits depicted in Fig. 2. To this end, we resort to the harmonic inversion technique of cross-correlated periodic orbit sums [7], which is an extension of periodic orbit quantization by harmonic inversion introduced in [5]. Here, we only briefly review the basic ideas and refer the reader to the literature for details. For simplicity, though without loss of generality, we focus on billiard systems, where the shape of the orbits is independent of the energy $E=\hbar^{2} k^{2} / 2 m$, with $k$ the wave number, and the classical action of orbits reads $S=\hbar k s$, with $s$ the physical length of the orbit.

The starting point is to introduce a weighted density of states in terms of $k$

$$
\varrho_{\alpha \alpha^{\prime}}(k)=-\frac{1}{\pi} \operatorname{Im} g_{\alpha \alpha^{\prime}}(k)
$$

with

$$
g_{\alpha \alpha^{\prime}}(k)=\sum_{n} \frac{b_{\alpha n} b_{\alpha^{\prime} n}}{k-k_{n}+\mathrm{i} \epsilon}
$$

where $k_{n}$ is the wave number eigenvalue of eigenstate $|n\rangle$ and

$$
b_{\alpha n}=\left\langle n\left|\hat{A}_{\alpha}\right| n\right\rangle
$$

are the diagonal matrix elements of a chosen set of $N$ linearly independent operators $\hat{A}_{\alpha}, \alpha=1,2, \ldots, N$. The Fourier transform of (2) yields an $N \times N$ cross-correlated signal

$$
C_{\alpha \alpha^{\prime}}(s)=\frac{1}{2 \pi} \int_{-\infty}^{+\infty} g_{\alpha \alpha^{\prime}}(k) \mathrm{e}^{-\mathrm{i} s k} \mathrm{~d} k=-\mathrm{i} \sum_{n} b_{\alpha n} b_{\alpha^{\prime} n} \mathrm{e}^{-\mathrm{i} k_{n} s} .
$$


A semiclassical approximation to the cross-correlated signal (4) has been derived in $[13,14]$. The cross-correlated periodic orbit signal reads

$$
C_{\alpha \alpha^{\prime}}^{\mathrm{sc}}(s)=-\mathrm{i} \sum_{\text {po }} \frac{a_{\alpha, \mathrm{po}} a_{\alpha^{\prime}, \mathrm{po}} s_{\mathrm{po}} \mathrm{e}^{-\mathrm{i} \frac{\pi}{2} \mu_{\mathrm{po}}}}{r\left|\operatorname{det}\left(M_{\mathrm{po}}-1\right)\right|^{1 / 2}} \delta\left(s-s_{\mathrm{po}}\right),
$$

where $r$ is the repetition number counting the traversals of the primitive orbit, and $M_{\mathrm{po}}$ and $\mu_{\mathrm{po}}$ are the monodromy matrix and Maslov index of the orbit, respectively. The weight factors $a_{\alpha, \text { po }}$ are classical averages over the periodic orbits

$$
a_{\alpha, \text { po }}=\frac{1}{s_{\mathrm{po}}} \int_{0}^{s_{\mathrm{po}}} A_{\alpha}(\mathbf{q}(s), \mathbf{p}(s)) \mathrm{d} s,
$$

with $A_{\alpha}(\mathbf{q}, \mathbf{p})$ the Wigner transform of the operator $\hat{A}_{\alpha}$. Semiclassical approximations to the eigenvalues $k_{n}$ and eventually also to the diagonal matrix elements $\left\langle n\left|\hat{A}_{\alpha}\right| n\right\rangle$ are now obtained by adjusting the semiclassical crosscorrelated periodic orbit signal (5) to the functional form of the quantum signal (4). The numerical tool for this procedure is an extension of the harmonic inversion method to the signal processing of cross-correlation functions $[15,16]$.

The resolution of the results depends on the signal length. For a one-dimensional signal, the method requires a signal length of $s_{\max } \approx 4 \pi \bar{\rho}(k)$, with $\bar{\rho}(k)$ the average density of states to resolve the eigenvalues [5]. This means that all periodic orbits up to the scaled action $s_{\max }$ have to be included. The advantage of using the cross-correlation approach is based on the realization that the total amount of independent information contained in the $N \times N$ signal is $N(N+1)$ multiplied by the length of the signal, while the total number of unknowns (here $b_{\alpha n}$ and $\left.k_{n}\right)$ is $(N+1)$ times the total number of poles $k_{n}$. Therefore the informational content of the $N \times N$ signal per unknown parameter is increased (as compared to the one-dimensional signal) by roughly a factor of $N$, and the cross-correlation approach should lead to a significant improvement of the resolution. The technique has been successfully applied in the periodic orbit quantization of an integrable system [7]. We will now demonstrate the power of the method in the semiclassical quantization of the (chaotic) closed three-disk system. As in Ref. [12], we concentrate on the quantum states with $A_{1}$ symmetry of the $C_{3 v}$ group.

The semiclassical cross-correlation signal (5) is constructed using the set of about 5 million periodic orbits represented in Fig. 2. We first construct a one-dimensional signal $(N=1)$ by simply choosing $\hat{A}_{1}=\mathbf{1}$, i.e., the unity operator. Fig. 3 contains the results for the eigenvalues $k_{n}$ of the closed threedisk system in terms of the corresponding energy values $E_{n}=k_{n}^{2} / 2$. The 


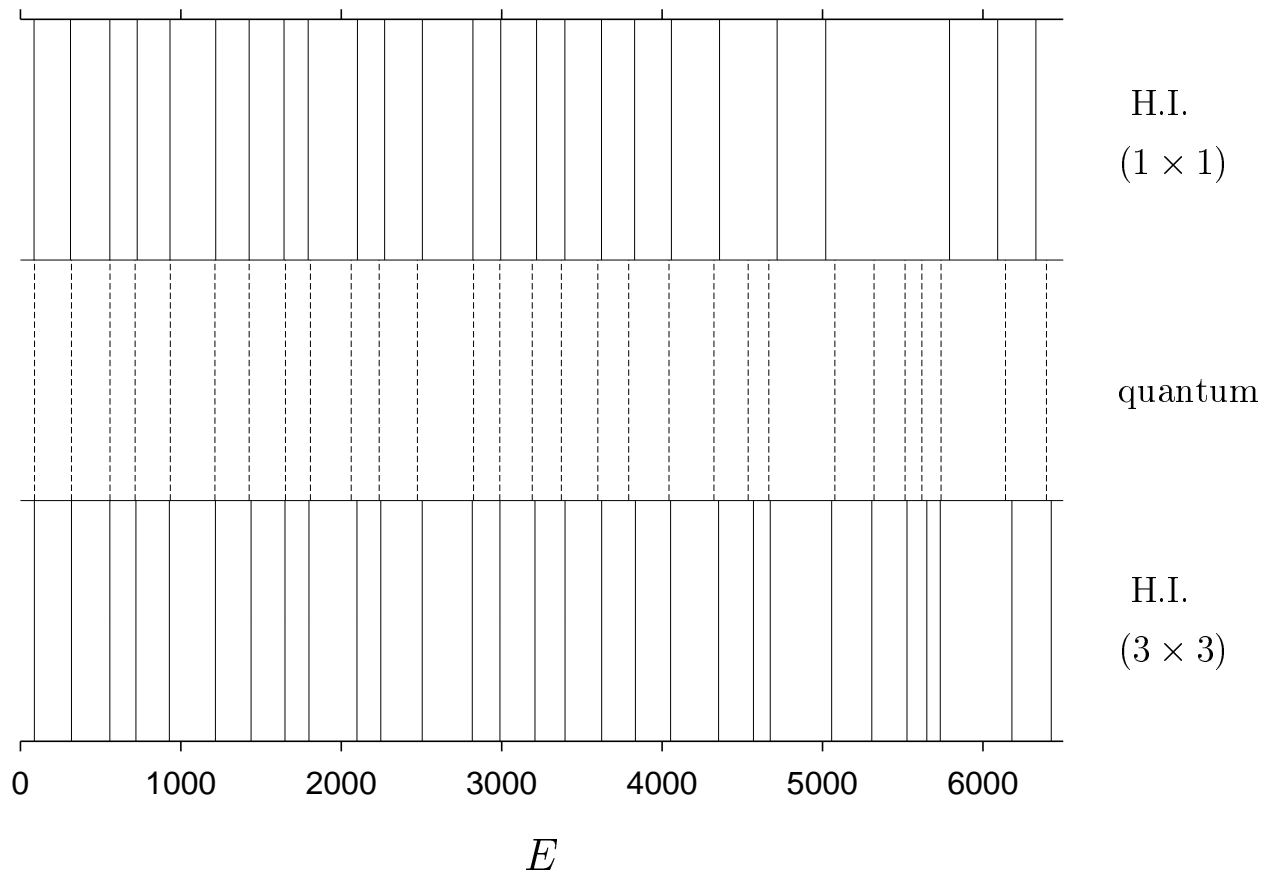

Fig. 3. Energy eigenvalues of the closed three-disk system. Top spectrum: Harmonic inversion of a one-dimensional periodic orbit signal of length $s_{\max }=4.9$. Middle spectrum: Exact quantum states (from [17]). Bottom spectrum: Semiclassical eigenvalues obtained by harmonic inversion of a $3 \times 3$ cross-correlated periodic orbit signal of length $s_{\max }=4.8$. The operators used to build the signal were $\mathbf{1}$ (unity), $r^{4}$, and $L^{4}$. The 29 lowest eigenvalues are well reproduced by semiclassical quantization.

uppermost spectrum is the result from harmonic inversion obtained from the one-dimensional signal of length $s_{\max }=4.9$. The spectrum in the middle shows the exact quantum results, taken from Ref. [17], for comparison. It can be seen that the lowest 20 eigenvalues are well reproduced by the results of the harmonic inversion of the $1 \times 1$ signal. The major part of the small deviations is due to the error inherent in the semiclassical approximation. However, it can also be seen that the signal length is not sufficient to resolve the higher eigenvalues, with energies $E \gtrsim 4500$, where the mean density of states with respect to $k$ (which grows $\sim \sqrt{E}$ ) is too large.

A further increase of the signal length in an attempt to resolve more eigenvalues is clearly inhibited by the rapid proliferation of periodic orbits in this system (cf. Fig. 2). Instead, we now exploit the cross-correlation technique to significantly improve the resolution. We construct the cross-correlated periodic orbit signal (5) using a set of three independent operators, viz. the operators $\hat{A}_{1}=1$ (unity), $\hat{A}_{2}=r^{4}$, and $\hat{A}_{3}=L^{4}$, with $r$ and $L$ the distance from the center of the billiard and the angular momentum, respectively. The bottom spectrum in Fig. 3 shows the results for the eigenvalues of the closed three-disk system obtained from the harmonic inversion of the $3 \times 3$ 
set of cross-correlated periodic orbit signals. Only the converged frequencies are presented, which have been selected by the criterion that their amplitude (multiplicity) should be close to 1 and the imaginary part of the frequencies close to zero (as the eigenvalues must be real). With the cross-correlated signal, all eigenvalues up to $E \lesssim 6500$ (a total of 29 levels) can be well reproduced.

The accuracy of the semiclassical results presented in Fig. 3 may at first glance seem surprising when one recalls the fact that the set of periodic orbits (see Fig. 2) used for constructing the signal (5), although being huge, is not strictly complete. For example, the channel marked (C) in Fig. 2 was cut off by restricting the number of successive leading zeros in the symbolic code. However, the Maslov indices of adjacent orbits in that channel differ by 2 , and therefore the periodic orbit amplitudes alternate in sign, which leads to approximate cancellations of terms in the periodic orbit signal. Furthermore, very unstable orbits with stability eigenvalues $|\lambda|>\lambda_{\max }=10^{9}$ were ignored in the analysis. By lowering the limit $\lambda_{\max }$ we have checked that indeed the very unstable orbits do not carry important information about the semiclassical spectrum, i.e., the results change only insignificantly. On the other hand, increasing $\lambda_{\max }$, and thus the total number of orbits used for harmonic inversion, will still slightly improve the accuracy of the semiclassical spectrum.

In conclusion, we have successfully carried out a "pure" periodic orbit quantization of a chaotic system with extremely rapid proliferation and strong pruning of orbits. We have demonstrated that harmonic inversion of crosscorrelation signals is indeed a powerful method for periodic orbit quantization, allowing the numerically stable handling even of huge periodic orbit sets. It should also be stressed that the semiclassical eigenvalues were obtained without having to resort to the mean density of states, which is difficult to calculate, including all necessary correction terms, even in the case of the closed three-disk system [17]. The present results may stimulate future work on other challenging systems without special structural information on the quantum or classical level. One example with strong pruning of the symbolic dynamics is the three-dimensional generalization of the closed three-disk billiard, i.e., a system consisting of four touching spheres at the corners of a regular tetrahedron. By contrast to the closed three-disk system this is an open system where no functional equation can be applied.

\section{Acknowledgments}

We thank A. Wirzba for communicating to us quantum mechanical data of the closed three-disk system. This work was supported by the Deutsche Forschungsgemeinschaft and Deutscher Akademischer Austauschdienst. 


\section{References}

[1] M. C. Gutzwiller, Chaos in Classical and Quantum Mechanics, Springer, New York, 1990.

[2] P. Cvitanović, B. Eckhardt, Phys. Rev. Lett. 63 (1989) 823.

[3] R. Aurich, C. Matthies, M. Sieber, F. Steiner, Phys. Rev. Lett. 68 (1992) 1629.

[4] M. V. Berry, J. P. Keating, J. Phys. A 23 (1990) 4839.

[5] J. Main, V. A. Mandelshtam, H. S. Taylor, Phys. Rev. Lett. 79 (1997) 825; J. Main, V. A. Mandelshtam, G. Wunner, H. S. Taylor, Nonlinearity 11 (1998) 1015 .

[6] J. Main, Phys. Rep. 316 (1999) 233.

[7] J. Main, K. Weibert, V. A. Mandelshtam, G. Wunner, Phys. Rev. E 60 (1999) 1639 .

[8] B. Eckhardt, G. Russberg, Phys. Rev. E 47 (1993) 1578.

[9] B. Eckhardt, G. Russberg, P. Cvitanović, P. E. Rosenqvist, P. Scherer, in: G. Casati, B. V. Chirikov (Eds.), Quantum Chaos, Cambridge University Press, Cambridge, 1995, p. 405.

[10] A. Wirzba, Phys. Rep. 309 (1999) 1.

[11] K. T. Hansen, Nonlinearity 6 (1993) 753.

[12] G. Tanner, P. Scherer, E. B. Bogomolny, B. Eckhardt, D. Wintgen, Phys. Rev. Lett. 67 (1991) 2410.

[13] J. Main, G. Wunner, Phys. Rev. E 60 (1999) 1630.

[14] S. Hortikar, M. Srednicki, Phys. Rev. E 61 (2000) R2180.

[15] E. Narevicius, D. Neuhauser, H. J. Korsch, N. Moiseyev, Chem. Phys. Lett. 276 (1997) 250.

[16] V. A. Mandelshtam, J. Chem. Phys. 108 (1998) 9999.

[17] P. Scherer, Ph.D. thesis, Universität Köln (1991). 